\title{
Profinite completions and Kazhdan's property (T)
}

\author{
Menny Aka
}

\begin{abstract}
We show that property (T) is not profinite, that is, we construct two finitely generated residually finite groups which have isomorphic profinite completions, while one admits property (T) and the other does not. This settles a question raised by M. Kassabov.
\end{abstract}

Mathematics Subject Classification (2010). 20E18, 22E40, 11H55.

Keywords. Profinite groups, profinite properties, Kazhdan's property (T), arithmetic groups.

\section{Introduction}

Two finitely generated groups have isomorphic profinite completions if and only if they have the same collection of finite quotients.

A property $\mathcal{P}$ of finitely generated residually finite groups is called a profinite property if the following is satisfied: if $\Gamma_{1}$ and $\Gamma_{2}$ are such groups with $\widehat{\Gamma_{1}} \cong \widehat{\Gamma_{2}}$ (i.e., the profinite completion of $\Gamma_{1}$ and $\Gamma_{2}$ are isomorphic) then $\Gamma_{1}$ has $\mathcal{P}$ if and only if $\Gamma_{2}$ has $\mathcal{P}$.

There are various interesting properties which are trivially profinite properties: e.g., having infinite Abelanization, rate of subgroup growth, etc. But there are other profinite properties which are in some sense less trivial, for example, having polynomial word growth (this follows from Gromov's seminal result on polynomial growth) and, for finitely presented groups, the property of being large (a group is said to be large if one of its finite index subgroups has a free non-Abelian quotient) [Lac07]. On the other hand, various other properties are not profinite. In a recent work ([CBW09]), it is shown that there exist two finitely presented residually-finite groups such that one is conjugacy separable and the other is not, and yet they have isomorphic profinite completions; this shows that being conjugacy separable is not a profinite property. In a work in preparation, Kassabov showed that it follows from [Kas07], [KN06] that the property $(\tau)$ is not a profinite property, and he asked whether the Kazhdan property $(\mathrm{T})$ is a profinite property. In this note, we show that the Kazhdan property (T) is not a profinite property. Explicitly we prove: 
Theorem 1. Let $D$ be a positive square-free integer, $k:=\mathbb{Q}(\sqrt{D})$ and $\mathcal{O}_{k}$ its ring of integers. Fix an integer $n \geq 6$ and let $\Gamma=\operatorname{Spin}(1, n)\left(\mathcal{O}_{k}\right)$ and $\Lambda=$ $\operatorname{Spin}(5, n-4)\left(\mathcal{O}_{k}\right)$. Then there exist finite-index subgroups $\Gamma_{0}<\Gamma$ and $\Lambda_{0}<\Lambda$ such that the profinite completion of $\Gamma_{0}$ is isomorphic to the profinite completion of $\Lambda_{0}$, while $\Lambda_{0}$ admits property $(T)$ and $\Gamma_{0}$ does not. Therefore, Kazhdan's property (T) is not profinite.

In particular, there exist non-isomorphic arithmetic groups with isomorphic profinite completions. Note that $\operatorname{Spin}(1, n)\left(\mathcal{O}_{k}\right)$ (resp. $\left.\operatorname{Spin}(5, n-4)\left(\mathcal{O}_{k}\right)\right)$ are central extensions of irreducible lattices in $H_{1}:=\mathrm{SO}(1, n) \times \mathrm{SO}(1, n)\left(\right.$ resp. $H_{2}:=$ $\mathrm{SO}(5, n-4) \times \mathrm{SO}(5, n-4))$. We can therefore deduce that $H_{1}$ and $H_{2}$ have lattices $\Gamma_{1}$ and $\Lambda_{1}$ resp. with isomorphic profinite completions. As $\mathbb{R}-\operatorname{rank}\left(H_{1}\right)=2$, while $\mathbb{R}-\operatorname{rank}\left(H_{2}\right)=10$ we see that the rank of the ambient Lie group is also not a profinite property (see also Remark 3.1 and compare with [PR72]).

While we can construct many non-trivial examples of non-isomorphic arithmetic groups with isomorphic profinite completions as the one above, we show in [Aka12] that every set of higher rank arithmetic groups with isomorphic profinite completion consists of finitely many isomorphism classes.

Our construction is based on a very simple idea. Vaguely speaking, the groups above have the congruence subgroup property and hence their profinite completions are essentially a product of the $p$-adic completions. Their $p$-adic completions agree since the quadratic forms $(1, n)$ and $(5, n-4)$ agree in every local field. Nevertheless, they do not agree over $\mathbb{R}$. Moreover, $\Gamma_{0}$ is a lattice in $\operatorname{Spin}(1, n) \times \operatorname{Spin}(1, n)$ while $\Lambda_{0}$ is a lattice in $\operatorname{Spin}(5, n-4) \times \operatorname{Spin}(5, n-4)$. The latter has $(\mathrm{T})$ while the former does not.

We also note that both groups admit property $\tau$.

This note is organized as follows: Much of our construction relies on quadratic forms theory, their Clifford algebra and their spin groups, so we review the relevant results and definitions in $\S 2$ together with a basic lemma on profinite completions. In $\S 3$ we prove Theorem 1 .

Acknowledgments. I would like to thank my advisor, Alex Lubotzky, for suggesting the problem and for reading several versions of this note. I would also like to thank Andrei Rapinchuk and Uzi Vishne for help and references on quadratic forms theory and their spin groups. Finally, I'd like to thank Martin Kassabov and the referee for their valuable comments.

This note is a part of the author's Ph.D. thesis at the Hebrew University. This research was partially funded by the "Hoffman Leadership and Responsibility" fellowship program, at the Hebrew University. I also thank the ISEF and the ERC for their support. 


\section{Preliminaries}

2.1. Quadratic forms and their spin groups. We denote the ring of $p$-adic integers by $\mathbb{Z}_{p}$.

Lemma 2. The quadratic forms

$$
q_{1}:=x_{1}^{2}+x_{2}^{2}+x_{3}^{2}+x_{4}^{2} \text { and } q_{2}:=-q_{1}=-x_{1}^{2}-x_{2}^{2}-x_{3}^{2}-x_{4}^{2}
$$

are equivalent over $\mathbb{Z}_{p}$ for every prime $p$, that is, for each prime $p$ there exist $M_{p} \in \mathrm{GL}_{4}\left(\mathbb{Z}_{p}\right)$ such that $M_{p} M_{p}^{t}=-I$.

Proof. We first note that for any $p$, the equation

$$
x_{1}^{2}+x_{2}^{2}+x_{3}^{2}+x_{4}^{2}=-1
$$

has solutions $\left(x_{p}, y_{p}, z_{p}, w_{p}\right) \in \mathbb{Z}_{p}^{4}$. Indeed, for $p \neq 2$, this follows from an easy application of Hensel's lemma together with the fact that in $\mathbb{F}_{p}$ each element is a sum to two squares. For $p=2$, we may take $\left(x_{2}, y_{2}, z_{2}, w_{2}\right)=(2,1,1, \sqrt{-7})$. Now, for each $p$, consider the matrix

$$
M_{p}:=\left(\begin{array}{rrrr}
x_{p} & y_{p} & z_{p} & w_{p} \\
-y_{p} & x_{p} & -w_{p} & z_{p} \\
-z_{p} & w_{p} & x_{p} & -y_{p} \\
-w_{p} & -z_{p} & y_{p} & x_{p}
\end{array}\right)
$$

and note that $M_{p} \in M_{4}\left(\mathbb{Z}_{p}\right), M_{p} M_{p}^{t}=-I$. This shows that $q_{1}$ and $q_{2}$ are equivalent over $\mathbb{Z}_{p}$ for all primes $p$.

Corollary 3. Let $q_{m, n}=\sum_{i=1}^{m} x_{i}^{2}-\sum_{i=1}^{n} y_{i}^{2}$. For $m \geq 4$, the quadratic form $q_{m, n}$ and $q_{m-4, n+4}$ are integrally equivalent over $\mathbb{Z}_{p}$ for all primes $p$. It follows that for any number field $k$ and a finite place $v$, we have that $q_{m, n}$ and $q_{m-4, n+4}$ are equivalent over $\mathcal{O}_{v}$, where $\mathcal{O}_{v}$ is the ring of integers of $k_{v}$.

Proof. Let $V_{i}$ be the quadratic space associated to $q_{i}, i=1,2$, and $V_{m, n}$ the quadratic space associated to $q_{m, n}$. Then for $m \geq 4$,

$$
V_{m, n} \cong V_{1} \oplus V_{m-4, n} \quad \text { and } \quad V_{m-4, n+4} \cong V_{2} \oplus V_{m-4, n}
$$

as quadratic spaces. Since $V_{1} \cong V_{2}$ over $\mathbb{Z}_{p}$ for all $p$, this holds for $V_{m, n}$ and $V_{m-4, n+4}$ as well.

In order to fix our notation and to fix specific representations of the groups involved, we recall the relevant definitions and give an explicit definition of the Clifford algebra and the spin group of a quadratic form. All objects and results that are described here can be found in [Cas78] (in particular, Chapter 10, §2) or in [FH91], $\S 20$. 
Given a non-degenerate quadratic space $(V, \phi)$ of dimension $n$ over a field $k$, there exists an associative algebra $C(V, \phi)$ over $k$ that contains $V$ as a linear subspace and satisfies:

(1) $C(V, \phi)$ is of dimension $2^{n}$ as a vector space.

(2) For all $x \in V, x \cdot x=\phi(x)$.

(3) $C(V, \phi)$ is generated as an algebra by $V$.

Moreover, these properties determine $C(V, \phi)$ uniquely (up-to a $k$-algebra isomorphism that fix $V$ ), and it is called the Clifford algebra of $V$.

We now give an explicit description of $C(V, \phi)$. This description depends on a choice of a normal basis, that is, a basis which is orthogonal with respect to the bilinear form associated to the quadratic form (see [Cas78], Chapter 10, §2).

Every non-degenerate quadratic space admits a normal basis and we fix a normal basis $e_{1}, \ldots, e_{n}$ for $(V, \phi)$. Recall that $C(V, \phi)$ contains $V$ and let $J$ be any subset of $\{1,2, \ldots, n\}$ arranged in ascending order, say

$$
j_{1}<j_{2}<\cdots<j_{r},
$$

where $r \leq n$, let

$$
e(J):=e_{j_{1}} \cdot e_{j_{2}} \cdots e_{j_{r}}
$$

be the multiplication of the $e_{j}$ 's, and let $e(\emptyset)$ be the unit element. Then the set

$$
\{e(J) \mid J \subset\{1,2, \ldots, n\} \text { ordered in ascending order, } 0 \leq|J| \leq n\}
$$

is a basis of $C(V, \phi)$. As $C(V, \phi)$ is also generated by $V$, the multiplication in $C(V, \phi)$ is determined by

$$
e_{i} e_{i}=\phi\left(e_{i}\right)
$$

and for $i \neq j$,

$$
e_{i} e_{j}=-e_{j} e_{i} .
$$

The even Clifford algebra of $C(V, \phi)$ is the algebra $C^{0}(V, \phi)$ generated by $\{e(J) \mid$ $|J|$ is even $\}$. There exists an involution

$$
\text { ': } C(V, \phi) \rightarrow C(V, \phi)
$$

defined on the basis elements by $(e(J))^{\prime}=e_{j_{r}} \cdot e_{j_{r-1}} \cdots e_{j_{1}}$ when $J$ is as above, and extended linearly. Let $\left(C^{0}(V, \phi)\right)^{*}$ denote the group of invertible elements of $C^{0}(V, \phi)$ and define $\operatorname{Spin}(V, \phi)$ by

$$
\operatorname{Spin}(V, \phi):=\left\{x \in\left(C^{0}(V, \phi)\right)^{*} \mid x x^{\prime}=1, x V x^{\prime} \subseteq V\right\} .
$$

Via right multiplication we obtain a faithful irreducible linear representation $\operatorname{Spin}(V, \phi) \rightarrow \operatorname{GL}\left(C^{0}(V, \phi)\right)$. We endow $\operatorname{GL}\left(C^{0}(V, \phi)\right)$ with the structure that 
is induced from the basis $\{e(J)|| J \mid$ is even $\}$ of $C^{0}(V, \phi)$. This gives a representation $\operatorname{Spin}(V, \phi) \rightarrow \mathrm{GL}_{2^{n-1}}(\mathbb{C})$, and we identify $\operatorname{Spin}(V, \phi)$ with its image of this representation.

We now turn to the case of $(V, \phi)=\left(V_{m, n}, q_{m, n}\right)$. Let

$$
G_{m, n}=\operatorname{Spin}\left(V_{m, n}, q_{m, n}\right) \quad \text { and } \quad C_{m, n}=C\left(V_{m, n}, q_{m, n}\right) .
$$

We choose the basis $e_{1}, \ldots, e_{m}, e_{m+1}, \ldots e_{m+n}$ of $V_{m, n}$ satisfying

$$
q_{m, n}\left(e_{i}\right)=\left\{\begin{aligned}
1 & \text { if } 1 \leq i \leq m, \\
-1 & \text { if } m+1 \leq i \leq m+n,
\end{aligned}\right.
$$

which is normal.

Using this basis we get by the above construction a specific faithful irreducible representation $G_{m, n} \rightarrow \mathrm{GL}\left(C_{m, n}^{0}\right) \cong \mathrm{GL}_{2^{m+n-1}}(\mathbb{C})$ via right multiplication. The isomorphism $\mathrm{GL}\left(C_{m, n}^{0}\right) \cong \mathrm{GL}_{2^{m+n-1}}(\mathbb{C})$ depends on our specific choice of a normal basis and we fix this choice throughout. For any ring $R \subset \mathbb{C}$, we let $G_{m, n}(R):=$ $G_{m, n} \cap \mathrm{GL}_{2^{m+n-1}}(R)$ and call this group the $R$-points of $G_{m, n}$. We remark that the (conjugacy class of the) representation of $G_{m, n}$ is independent of the choice of a normal basis for the quadratic space, but the group of $R$-points, for a general ring $R$, may depend on this choice. For this reason, we fix throughout the above representations of $G_{m, n}$.

The group $G_{m, n}$ is known to be an almost simple and absolutely simple algebraic group defined over $\mathbb{Q}$. For any field $k \subset \mathbb{R}, \mathrm{k}-\operatorname{rank}\left(G_{m, n}\right)=\min (m, n)$ (see for example [FH91], §20). Moreover, we have the following consequence of Corollary 3 :

Corollary 4. Let $m>4, n>0$ be natural numbers, $k$ be any number field and $v a$ discrete valuation on $k$. Then, under the fixed representations which are described above, $G_{m, n}\left(\mathcal{O}_{v}\right)$ is isomorphic to $G_{m-4, n+4}\left(\mathcal{O}_{v}\right)$, where $\mathcal{O}_{v}$ denotes the ring of integers in the completion of $k$ with respect to $v$.

Proof. Using Corollary 3, this follows readily from the definitions in [Cas78], Chapter $10, \S 2$. Moreover, if we had defined these groups in the language of group schemes, this corollary would have been immediate. Nevertheless, since this is crucial for our construction, we give a complete proof, which is rather technical.

Let $m^{\prime}=m-4, n^{\prime}=n+4$, and let $k_{v}$ denote the completion of $k$ with respect to $v$. Let $\left(k_{v}^{m+n}, q_{m, n}\right)$ be the quadratic space associated $q_{m, n}$ with the standard normal basis $e_{1}, \ldots, e_{m+n}$, that is,

$$
q_{m, n}\left(e_{i}\right)=\left\{\begin{aligned}
1 & \text { if } 1 \leq i \leq m \\
-1 & \text { if } m+1 \leq i \leq m+n
\end{aligned}\right.
$$

By Corollary 3, there exists $M \in \mathrm{GL}_{m+n}\left(\mathbb{Z}_{p}\right)$ such that $\left\{f_{i}:=M e_{i}\right\}_{i=1}^{m+n}$ is also a 
normal basis of $\left(k_{v}^{m+n}, q_{m, n}\right)$ with

$$
q_{m, n}\left(f_{i}\right)=\left\{\begin{aligned}
1 & \text { if } 1 \leq i \leq m^{\prime}, \\
-1 & \text { if } m^{\prime}+1 \leq i \leq m^{\prime}+n^{\prime} .
\end{aligned}\right.
$$

Let $C:=C^{0}\left(k_{v}^{m+n}, q_{m, n}\right)$ and $C^{\prime}=C^{0}\left(k_{v}^{m^{\prime}+n^{\prime}}, q_{m^{\prime}, n^{\prime}}\right)$. We will show that $\mathrm{GL}(C)\left(\mathcal{O}_{v}\right) \cong \mathrm{GL}\left(C^{\prime}\right)\left(\mathcal{O}_{v}\right)$, and the corollary readily follows since $G_{m, n}\left(\mathcal{O}_{v}\right):=$ $G_{m, n} \cap \operatorname{GL}(C)\left(\mathcal{O}_{v}\right)$ (and resp. for $\left.G_{m^{\prime}, n^{\prime}}\right)$.

The construction above shows that the bases $\left\{e_{i}\right\}$ and $\left\{f_{i}\right\}$ give rise to different bases on $C$, which we denote by $E$ and $F$. The bases $E$ and $F$ gives rise to two structures on $\mathrm{GL}(C)$, i.e., two isomorphisms

$$
\Phi_{E}, \Phi_{F}: \mathrm{GL}(C) \rightarrow \mathrm{GL}_{2^{m+n-1}}(\mathbb{C}) .
$$

The base $E$ is the base fixed above, and by $\operatorname{GL}(C)\left(\mathcal{O}_{v}\right)$ we mean the $\mathcal{O}_{v}$-points of $\mathrm{GL}(C)$ with respect to the basis $E$, i.e., $\Phi_{E}^{-1}\left(\mathrm{GL}_{2^{m+n}}\left(\mathcal{O}_{v}\right)\right)$. By equation (1), $\mathrm{GL}\left(C^{\prime}\right)\left(\mathcal{O}_{v}\right)$ is isomorphic to the $\mathcal{O}_{v}$-points of $\mathrm{GL}(C)$ with respect to the basis $F$. So we may conclude by showing that the $\mathcal{O}_{v}$-points of $\operatorname{GL}(C)$ with respect to $E$ are isomorphic to the $\mathcal{O}_{v}$-points of $\mathrm{GL}(C)$ with respect to $F$.

Let $\tilde{M}$ denote the base change matrix from $E$ to $F$, which is called the derived matrix of $M$. From the multiplication rules in $C$ and the fact that $M$ has entries in $\mathbb{Z}_{p}$ and $q_{m, n}$ and $q_{m^{\prime}, n^{\prime}}$ has coefficients in $\mathbb{Z}_{p}$, it follows that $\widetilde{M}$ also has entries in $\mathbb{Z}_{p}$. The inverse of $\tilde{M}$ is the derived matrix of $M^{-1}$, which also has entries in $\mathbb{Z}_{p}$ by the same argument. Conjugation by $\tilde{M}$, which we denote by $\operatorname{Int}(\tilde{M})$, identifies the representations $\Phi_{E}, \Phi_{F}$, that is, $\operatorname{Int}(\tilde{M}) \circ \Phi_{E}=\Phi_{F}$. It follows that the $\mathcal{O}_{v}$-points of $\mathrm{GL}(C)$ with respect to $E$ and with respect to $F$ are isomorphic by $\operatorname{Int}(\tilde{M})$.

\subsection{A basic lemma on profinite completion}

Lemma 5. Let $\Gamma$ be a residually finite group. There is a one-to-one correspondence between the set $\mathcal{X}$ of all finite-index subgroups of $\Gamma$ and the set $y$ of all open subgroup of $\widehat{\Gamma}$, given by

$$
X \mapsto \bar{X} \quad(X \in \mathcal{X}), \quad Y \mapsto Y \cap \Gamma \quad(Y \in \mathcal{Y}),
$$

where $\bar{X}$ denotes the closure of $X$ in $\hat{\Gamma}$. Moreover, $\bar{X}$ is canonically isomorphic to $\hat{X}$ and

$$
[\Gamma: X]=[\widehat{\Gamma}: \hat{X}] .
$$

Proof. See [LS03], Proposition 16.4.3. 


\section{Property (T) is not profinite - proof of Theorem 1}

We continue with the notation of Theorem 1 and we let $G_{1}:=G_{n, 1}$ and $G_{2}:=$ $G_{n-4,5}$ with the fixed representations described above. Let $\sigma_{1}, \sigma_{2}$ be the two distinct embeddings of $k$ into $\mathbb{R}$. They induce natural embeddings $\hat{\sigma}_{1}, \hat{\sigma}_{2}$ of $G_{i}\left(\mathcal{O}_{k}\right)$ to $G_{i}(\mathbb{R})$. We embed

$$
\Gamma:=G_{1}\left(\mathcal{O}_{k}\right) \rightarrow G_{1}(\mathbb{R}) \times G_{1}(\mathbb{R}), \quad x \mapsto\left(\hat{\sigma}_{1}(x), \hat{\sigma}_{2}(x)\right),
$$

and similarly

$$
\Lambda:=G_{2}\left(\mathcal{O}_{k}\right) \rightarrow G_{2}(\mathbb{R}) \times G_{2}(\mathbb{R}), \quad x \mapsto\left(\hat{\sigma}_{1}(x), \hat{\sigma}_{2}(x)\right) .
$$

It is well known that these embeddings realize $\Gamma$ and $\Lambda$ as irreducible lattices.

Now we show that any finite-index subgroup $\Gamma_{0}<\Gamma$ does not have property (T), while any finite-index subgroup $\Lambda_{0}<\Lambda$ does. The group $G_{1}(\mathbb{R})=\operatorname{Spin}(n, 1)(\mathbb{R})$, which is a central extension of $\mathrm{SO}(n, 1)(\mathbb{R})$, does not have property (T) ([BdlHV08], Theorem 3.5.4) and neither does the direct product of $G_{1}(\mathbb{R})$ with itself ([BdlHV08], Proposition 1.7.8). Note that any finite-index subgroup $\Gamma_{0}<\Gamma$ is a lattice in $G_{1}(\mathbb{R}) \times$ $G_{1}(\mathbb{R})$. Since a lattice in a group has property (T) if and only if the group has property (T) ([BdlHV08], Proposition 1.7.1), it follows that any finite-index subgroup $\Gamma_{0}<\Gamma$ does not have property (T).

In contrast, the group $G_{2}(\mathbb{R})=\operatorname{Spin}(n-4,5)(\mathbb{R})$, which is a central extension of $\mathrm{SO}(n-4,5)(\mathbb{R})$, does have property $(\mathrm{T})$ since it is almost simple of rank $\geq 2$ and therefore so does its direct product with itself ([BdlHV08], Proposition 1.7.8). Again, any finite-index subgroup $\Lambda_{0}<\Lambda$ is a lattice in $G_{2}(\mathbb{R}) \times G_{2}(\mathbb{R})$. It follows that any finite-index subgroup $\Lambda_{0}<\Lambda$ has property (T). In particular, a finite-index subgroup of $\Gamma$ cannot be isomorphic to a finite-index subgroup of $\Lambda$.

Nevertheless, we will now show that there exist a finite-index subgroup $\Gamma_{0}<\Gamma$ and a finite-index subgroup $\Lambda_{0}<\Lambda$ that have isomorphic profinite completions. First note that, by Corollary $4, G_{1}\left(\mathcal{O}_{v}\right) \cong G_{2}\left(\mathcal{O}_{v}\right)$ for any discrete valuation $v$, so there exists an isomorphism

$$
\Phi: \prod_{v} G_{1}\left(\mathcal{O}_{v}\right) \rightarrow \prod_{v} G_{2}\left(\mathcal{O}_{v}\right),
$$

where the product runs over all the discrete valuations of $k$.

By [Kne79], 11.3, the congruence kernel of $G_{2}$ is the trivial group and therefore

$$
\widehat{\Lambda}=\widehat{G_{2}\left(\mathcal{O}_{k}\right)} \cong G_{2}\left(\widehat{\mathcal{O}}_{k}\right)=\prod_{v} G_{2}\left(\mathcal{O}_{v}\right),
$$

where the product runs over all discrete valuations of $k$. For $G_{1}$, by [Kne79], 11.5c, the congruence kernel is of size 1 or 2 . In any case, it is finite, so $\hat{\Gamma}$ fits into the following short exact sequence

$$
1 \rightarrow C \rightarrow \hat{\Gamma} \rightarrow \prod_{v} G_{1}\left(\mathcal{O}_{v}\right) \rightarrow 1,
$$

where $C$ is a finite group. 
As $\widehat{\Gamma}$ is profinite, we can find a finite-index open subgroup that intersects $C$ trivially and therefore maps to $\prod_{v} G_{1}\left(\mathcal{O}_{v}\right)$ injectively. By Lemma 5, this subgroup is necessarily of the form $\widehat{\Gamma_{0}}$, for a finite-index subgroup $\Gamma_{0}$ of $\Gamma$.

We identify $\widehat{\Gamma_{0}}$ with its (faithful) image in $\prod_{v} G_{1}\left(\mathcal{O}_{v}\right)$. By Lemma 5 , there exists a finite-index subgroup $\Lambda_{0}$ of $\Lambda$ with $\widehat{\Lambda_{0}} \cong \Phi\left(\widehat{\Gamma_{0}}\right)$, i.e., $\widehat{\Lambda_{0}}$ fits in the following commutative diagram:

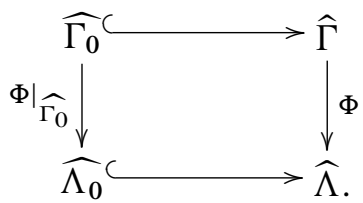

Thus $\widehat{\Lambda_{0}}$ and $\widehat{\Gamma_{0}}$ are isomorphic. As explained above, $\Lambda_{0}$ has property $(\mathrm{T})$ and $\Gamma_{0}$ does not. This concludes the proof of Theorem 1 .

3.1. Remark. The above proof also shows that the rank of the Lie group containing $\Gamma$ as a lattice is not a profinite property. Indeed, using Corollary 4 and induction, one sees that for arbitrarily large fixed $n$ and every $0 \leq k \leq \frac{n}{8}$, the groups $G_{k} \times G_{k}$ with $G_{k}:=\operatorname{Spin}(4 k+1, n-4 k)$ have irreducible lattices $\Gamma_{k}$ which share the same profinite completions but are pairwise non-isomorphic by Mostow's strong-rigidity (which may be applied to the images of $\Gamma_{k}$ in $\mathrm{SO}(4 k+1, n-4 k) \times \mathrm{SO}(4 k+1, n-4 k)$ ). The rank of $G_{k} \times G_{k}$ is $2(4 k+1)$.

\section{References}

[Aka12] M. Aka, Arithmetic groups with isomorphic finite quotients. J. Algebra 352 (2012), 322--340.

[BdlHV08] B. Bekka, P. de la Harpe, and A. Valette, Kazhdan's property (T). New Math. Monogr. 11, Cambridge University Press, Cambridge 2008. Zbl 1146.22009 MR 2415834

[Cas78] J. W. S. Cassels, Rational quadratic forms. London Math. Soc. Monogr. 13, Academic Press, London 1978. Zbl 0395.10029 MR 522835

[CBW09] O. Cotton-Barratt and H. Wilton, Conjugacy separability of 1-acylindrical graphs of free groups. Preprint 2009. arXiv:0906.0101

[FH91] W. Fulton and J. Harris, Representation theory. Graduate Texts in Math. 129, Springer-Verlag, New York 1991. Zbl 0744.22001 MR 1153249

[Kas07] M. Kassabov, Symmetric groups and expander graphs. Invent. Math. 170 (2007), 327-354. Zbl 1191.20002 MR 2342639

[KN06] M. Kassabov and N. Nikolov, Cartesian products as profinite completions. Internat. Math. Res. Notices 2006 (2006), Art. ID 72947. Zbl 1129.20019 MR 2264720

[Kne79] M. Kneser, Normalteiler ganzzahliger Spingruppen. J. Reine Angew. Math. 311/312 (1979), 191-214. Zbl 0409.20038 MR 549966 
[Lac07] M. Lackenby, Detecting large groups. J. Algebra 324 (2010), 2636-2657. Zbl 1231.20026 MR 2725193

[LS03] A. Lubotzky and D. Segal, Subgroup growth. Progr. Math. 212, Birkhäuser Verlag, Basel 2003. Zbl 1071.20033 MR 1978431

[PR72] G. Prasad and M. S. Raghunathan, Cartan subgroups and lattices in semi-simple groups. Ann. of Math. (2) 96 (1972), 296-317. Zbl 0245.22013 MR 0302822

Received February 23, 2010; revised February 2, 2011

M. Aka, Institute of Mathematics, The Hebrew University, Givat Ram, Jerusalem 91904, Israel

E-mail: mennyaka@math.huji.ac.il 